\title{
Physical and Mechanical Properties of Niobium for SRF Science and Technology
}

\author{
Ganapati Rao Myneni \\ Accelerator Division \\ Jefferson Lab \\ Newport News, Virginia, USA
}

\begin{abstract}
Optimized mechanical and physical properties of high purity niobium are crucial for obtaining high performance SRF particle beam accelerator structures consistently. This paper summarizes these important material properties for both high purity polycrystalline and single crystal niobium.
\end{abstract}

Keywords: Niobium, yield stress, tensile stress, ductility, Vickers hardness, thermal conductivity, residual resistance ratio, critical magnetic field, SRF cavity

PACS: 62.20.-x, 62.20.Fe, 65.40.0-b, 74.25.Ha, 74.70.ad

\section{INTRODUCTION}

High purity niobium is the material of choice for the SRF cavities used in particle accelerators. The SRF cavity grade niobium is either produced from pyrochlore ore (CBMM's Araxá, Brazil mine holds 90\% of world's deposits) or columbite/tantalite ore. The Jefferson Lab's CEBAF, the largest solid niobium SRF accelerator in the world, is built with CBMM pyrochlore polycrystalline niobium sheets with RRR > 250 [1, 2, 3]. The Spallation Neutron Source (SNS, Oakridge, Tennessee) SRF cavities are produced with columbite/tantalite polycrystalline niobium sheets. Generally, it is well known that the SRF cavities need to be annealed at high temperatures (up to $900{ }^{\circ} \mathrm{C}$ ) for degassing the hydrogen in order to maintain high quality factors and to minimize Q-disease problem [4, 5]. During the initial stages of the SNS cavity development, the polycrystalline high purity niobium cavities exhibited micro-yielding issues after annealing at $800{ }^{\circ} \mathrm{C}$ even for 1 hour (resulting in deformation and consequent non uniform field flatness of the cells) [6, 7]. Additionally, it was also found difficult to meet the yield strength specifications for high purity niobium sheets once they were fully recrystallized [8].

A Cooperative Research And Development Agreement (CRADA) is initiated by CBMM's subsidiary Reference Metals Company Inc. and Jefferson Lab in August 2004 to study the interstitial (H, C, N, O) interactions in niobium and to develop techniques for accurately determining the interstitial content in high purity niobium. This collaboration is also aimed to optimize the substitutional tantalum (Ta) impurity content in high purity niobium in order to consistently obtain high performance SRF cavities at reduced cost. As part of this CRADA program niobium ingot slices were 
received from CBMM. High ductility (100\% elongation) was measured during the initial mechanical property evaluation of the material from the ingot slices leading to the novel innovative idea of producing cavities from sliced ingot material [9, 10]. Since then large grain-single crystal niobium technology has evolved very quickly resulting in the organization of this single crystal niobium technology workshop at CBMM's Araxá mine in Brazil. In the mean time, the CRADA is extended for three more years (2007 - 2010). During this period we are aiming to optimize the physical and mechanical properties of high purity niobium to reduce the cost of SRF particle beam accelerator structures for $\mathrm{CW}$ and industrial applications.

In the following, the mechanical (yield strength, tensile strength, hardness and ductility) and physical properties (thermal conductivity-residual resistance ratio and DC critical magnetic field) of polycrystalline and single crystal high purity niobium are summarized.

\section{PHYSICAL AND MECHANICAL PROPERTIES}

\section{Polycrystalline Niobium}

Figures 1 to 4 show the average mechanical properties yield \& tensile strengths, ductility and Vickers hardness of fully recrystallized high purity polycrystalline niobium samples as a function of annealing temperature. The first data point at $20^{\circ} \mathrm{C}$ is for as received niobium samples, the $600^{\circ} \mathrm{C}$ data point is for the niobium samples annealed at $600{ }^{\circ} \mathrm{C}$ for 10 hours, the $800 \mathrm{C}$ data point relates to the niobium annealed at $800 \mathrm{C}$ for 6 hours and the $1250{ }^{\circ} \mathrm{C}$ data point is for samples annealed at $1250{ }^{\circ} \mathrm{C}$ for 6 hours in the presence of $\mathrm{Ti}$ solid state getter. All the samples were annealed in an UHV furnace.

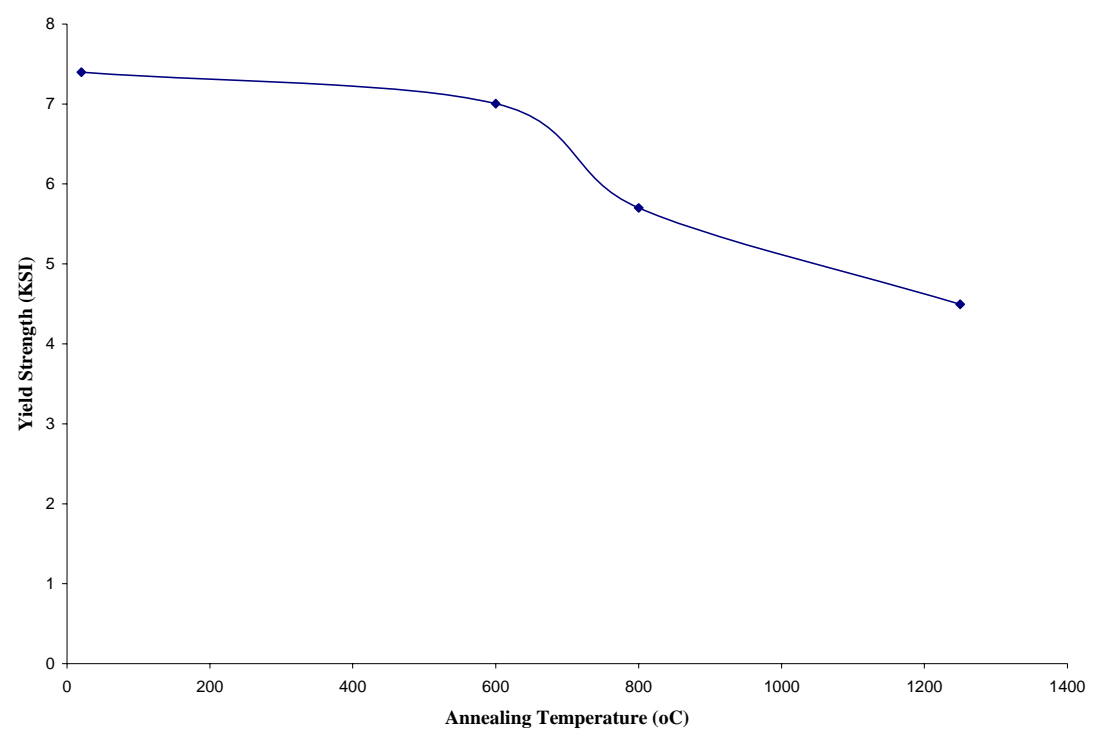

FIGURE 1. Yield strength of polycrystalline niobium as a function of annealing temperature 


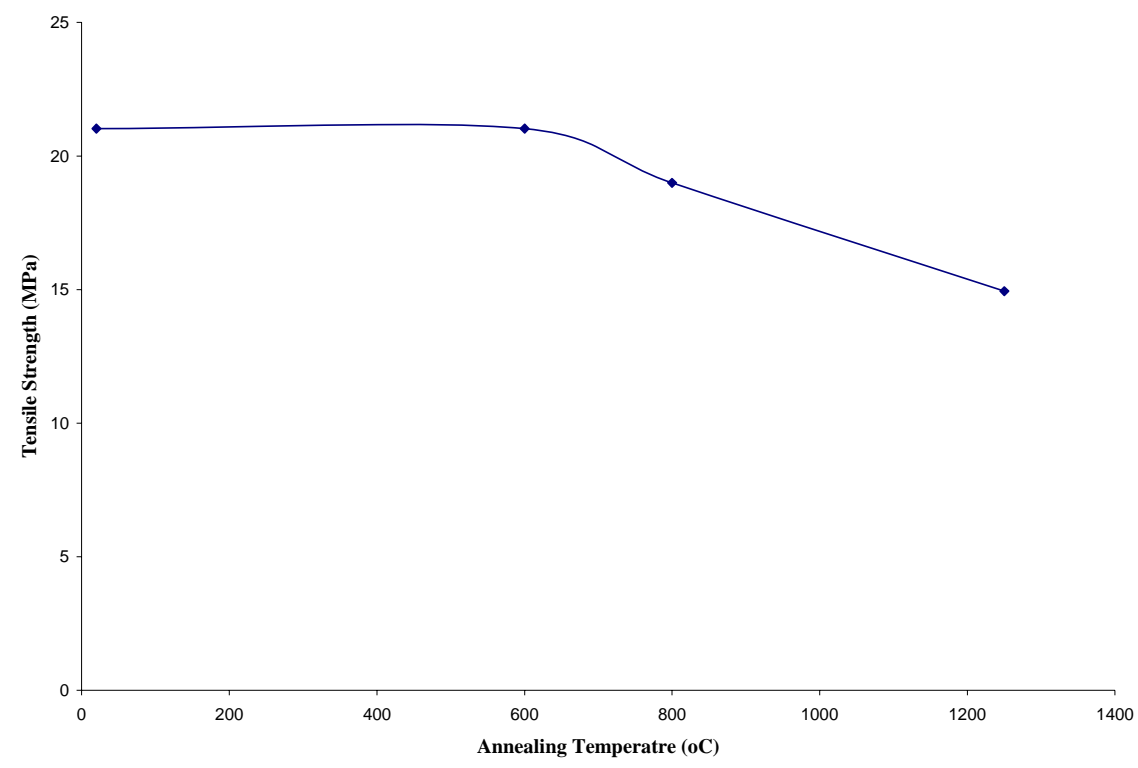

FIGURE 2. Tensile strength of polycrystalline niobium as a function of annealing temperature

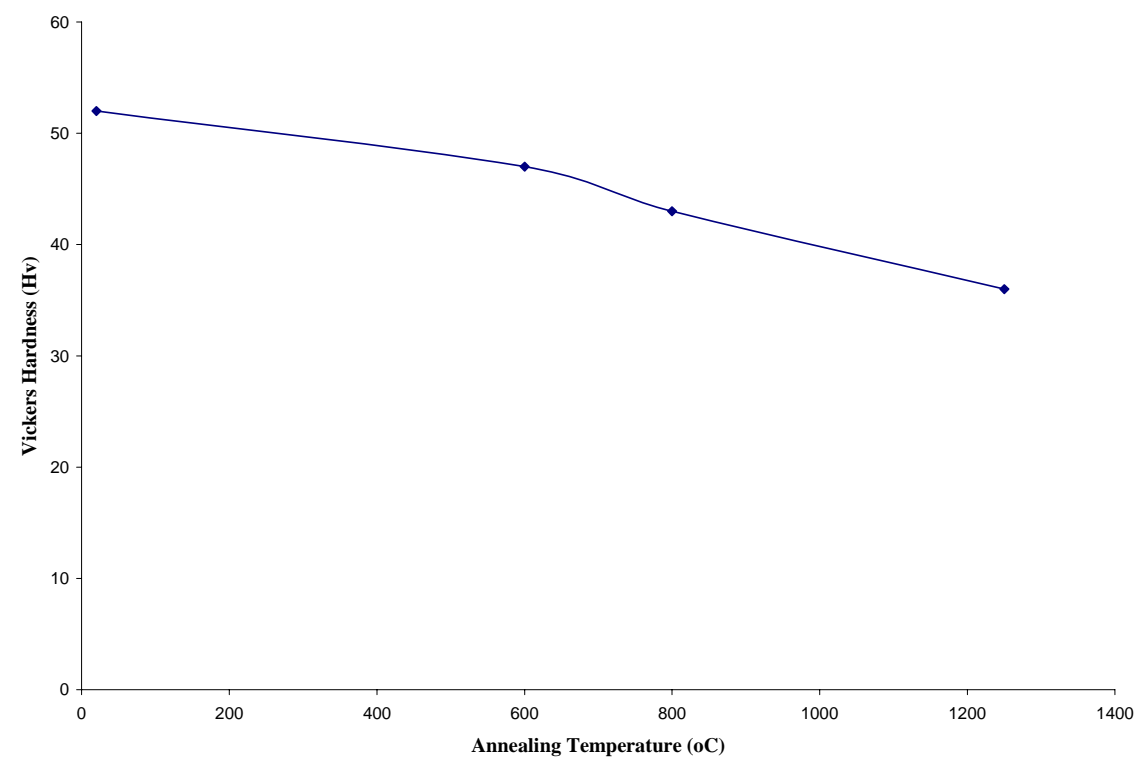

FIGURE 3. Vickers hardness of polycrystalline niobium as a function of annealing temperature 


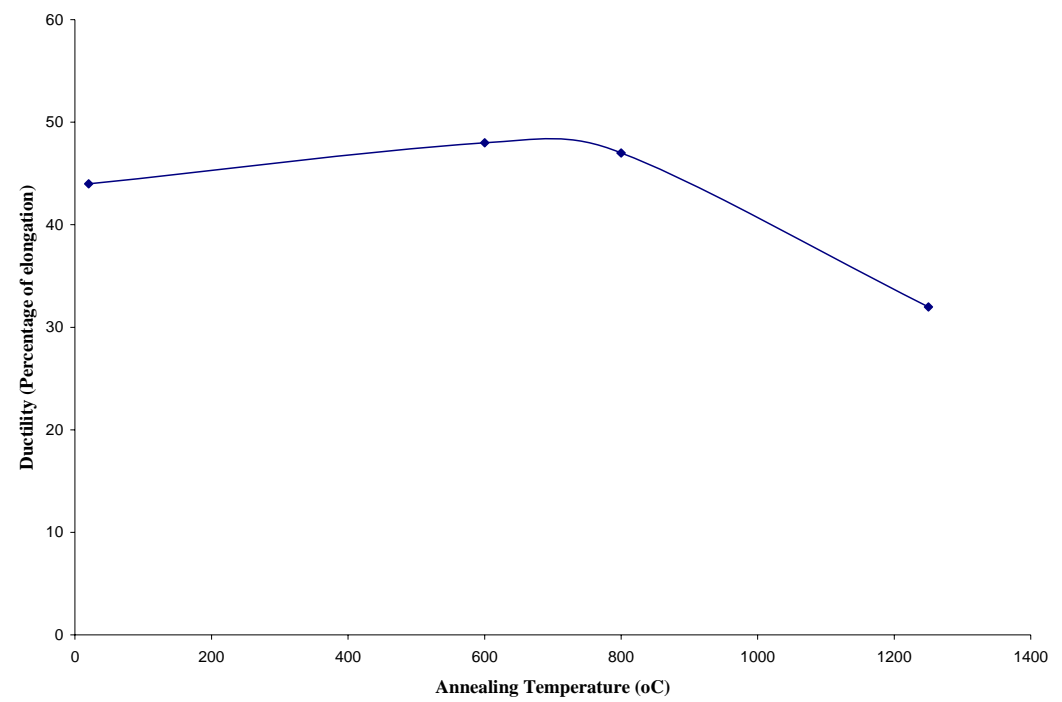

FIGURE 4. Ductility of polycrystalline niobium as a function of annealing temperature

The residual resistance ratio (RRR) of the polycrystalline niobium was estimated from the measured thermal conductivity of the samples at $4.2 \mathrm{~K}$ with the well known conversion factor of 4 resulting from the Wiedmann-Franz law and is shown in Figure 5 as a function of annealing temperature.

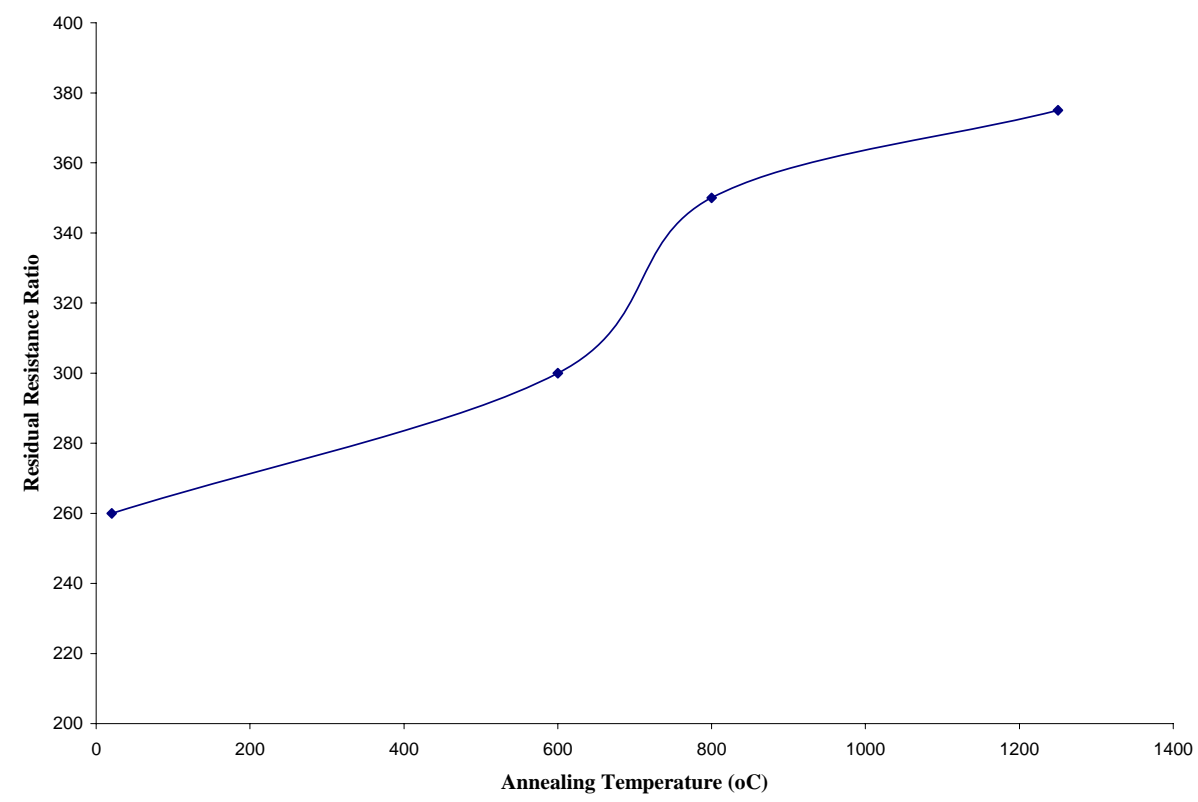

FIGURE 5. RRR of polycrystalline niobium as a function of annealing temperature 


\section{Single Crystal Niobium}

The mechanical properties of the single crystal niobium for pristine as received, BCPed ( 200 microns removed) and heat treated with Ti solid state getter at $1250{ }^{\circ} \mathrm{C}$ for 3 hours conditions are shown in Figure 6. The figure also presents the Vickers hardness data of the samples. The yield strength of the pristine single crystal niobium is 9.8 KSI, the yield strength of BCP'ed sample reduces to $5.5 \mathrm{KSI}$ and the yield strength of the heat treated sample further drops to $4.5 \mathrm{KSI}$. This drop in yield strength with BCP can be attributed to softening of the material from the absorbed hydrogen into niobium during the chemical etching. However, it is not clear why the yield strength further reduced with heat treatment at $1250{ }^{\circ} \mathrm{C}$ with $\mathrm{Ti}$ which can be expected to remove the dissolved hydrogen. It is likely that during the furnace cool down some of the hydrogen (pp of hydrogen in the furnace $\sim 10^{-6}$ Torr) might have been reabsorbed into more energetically favorable sites. As can be expected the tensile strength also reduced in line with the drop in yield strength. The softening effect is also indicated by the reduced Vickers hardness from 85 for the pristine sample to 44 for the BCPed and heat treated samples.

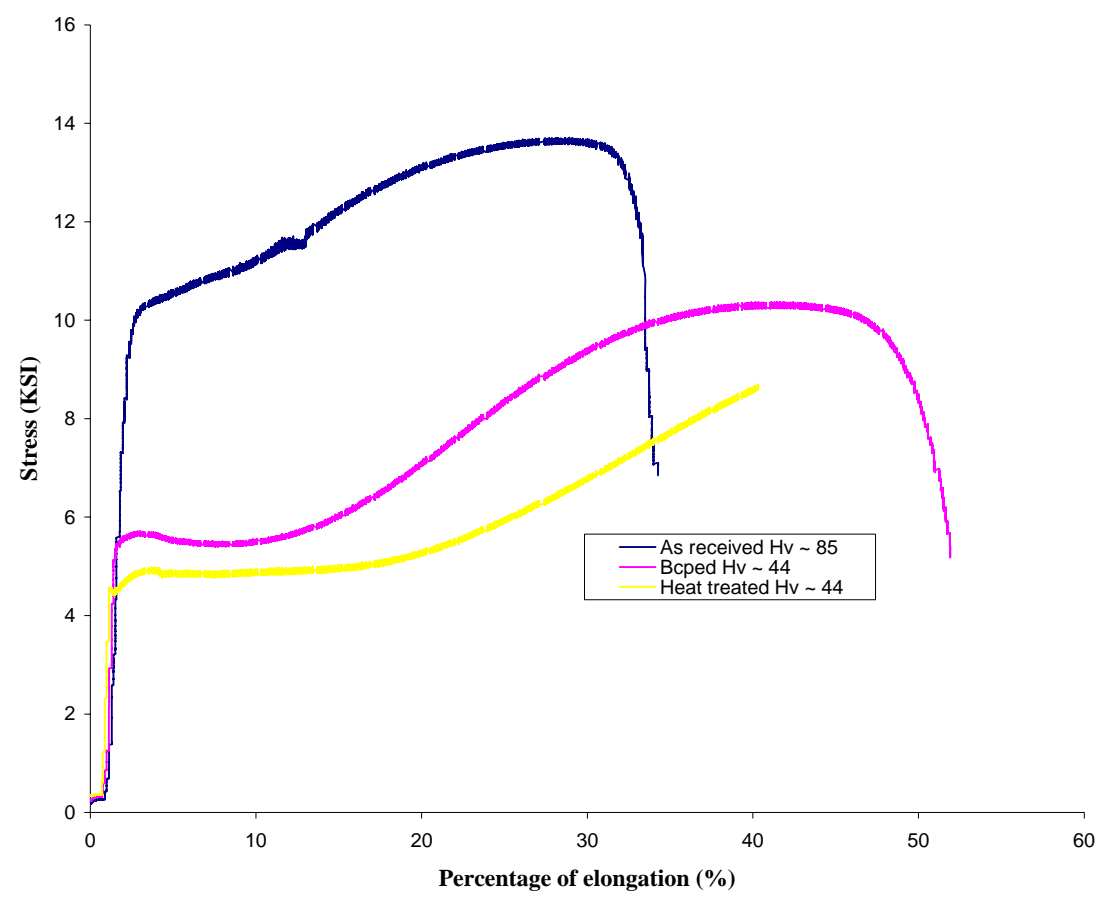

FIGURE 6. Mechanical properties of single crystal niobium with various treatments 
Thermal conductivity of polycrystalline (as received \& heat treated), heat treated single crystal and two crystal niobium is shown in Figure 7. The phonon peak can be seen only for the annealed single crystal niobium sample due to the elimination of defects by heat treatment. As expected the polycrystalline, two crystal and polycrystalline heat treated niobium samples did not exhibit the phonon peak due to the grain boundaries and defects [11].

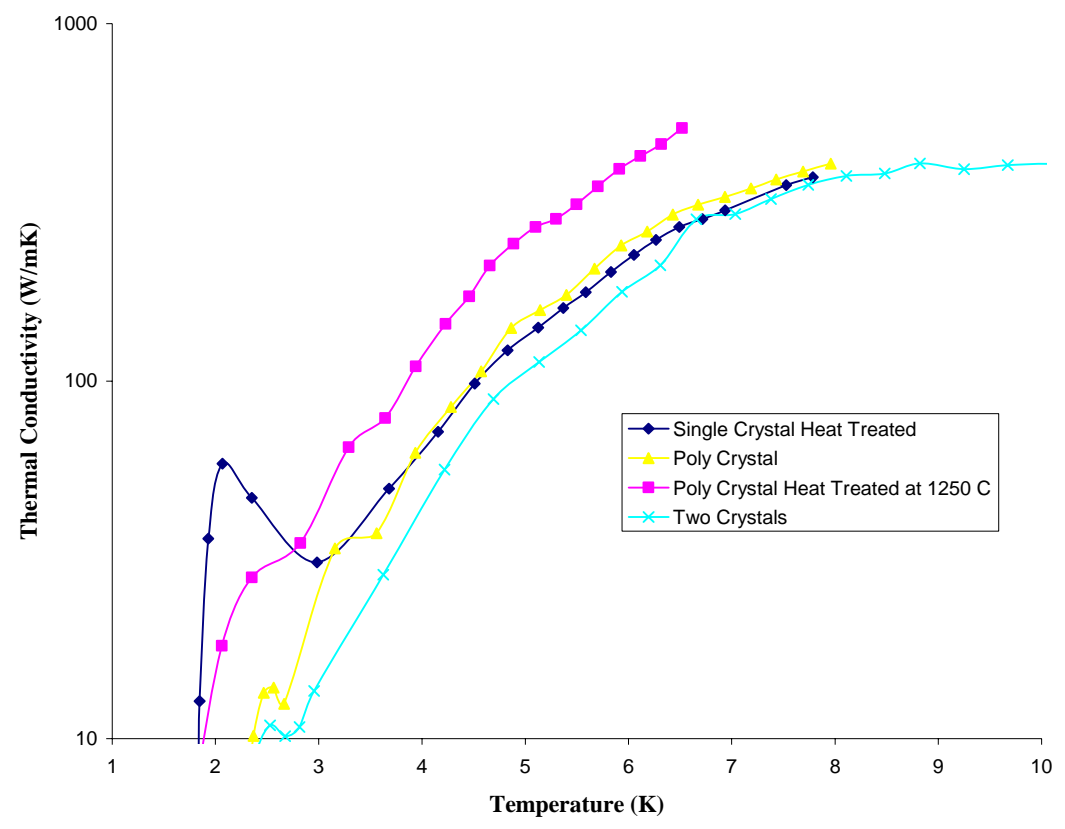

FIGURE 7. Thermal conductivity of polycrystalline and single crystal niobium

The most important physical property that has direct bearing on the performance characteristic of the SRF cavities is the critical magnetic field after the surface contamination issues are eliminated. RRCAT, India and JLab are presently investigating how the various treatments affects on the DC critical magnetic fields of polycrystalline and single crystal niobium with varying amounts of tantalum. The preliminary results indicate that at $2 \mathrm{~K}$ the pristine polycrystalline niobium's Hc1 is $180 \mathrm{mT}$ while Hc1 of the pristine single crystal niobium can be as high as $\sim 240 \mathrm{mT}$. Chemical treatments appear to suppress the critical magnetic filed considerably. Hydrogen is the only interstitial that is absorbed into niobium during chemical or heat treatments and may be responsible for the reduced critical magnetic fields. These results will be published in a peer reviewed journal in the very near future [12]. 


\section{SUMMARY}

This paper briefly summarizes the physical and mechanical properties of polycrystalline and single crystal SRF cavity grade niobium. Hydrogen is the main interstitial that changes with various treatments and it is expected to influence the performance of SRF cavities.

\section{Acknowledgments}

This manuscript has been authored by Jefferson Science Associates, LLC under U.S. DOE Contract No. DE-AC05-06OR23177. The U.S. Government retains a nonexclusive, paid-up, irrevocable, world-wide license to publish or reproduce this manuscript for U.S. Government purposes. This work is partially supported by Reference Metals Company Inc. under the joint CRADA SURA 2004 S002 and the author is grateful to Mr. Tadeu Carneiro of CBMM, Brazil for many useful discussions.

\section{REFERENCES}

1. R. A. Bosch, "Production of High RRR Niobium Discs for SURA/CEBAF" in the Proceedings of the Conference Electron Beam Melting and Refining State of the Art 1990, edited by R. Bakish, Bakish Materials Corporation, Englewood, NJ, 1990, pp. 189-207.

2. P. Kneisel, J. Mammosser, M. G. Rao, K. Saito and M. Peiniger, "Superconducting Cavities from High Thermal Conductivity Niobium for CEBAF” in the Proceedings of the Conference Electron Beam Melting and Refining State of the Art 1990, edited by R. Bakish, Bakish Materials Corporation, Englewood, NJ, 1990, pp. 177-188.

3. M. G. Rao and P. Kneisel, "Mechanical Properties of High RRR Niobium at Cryogenic Temperatures” in Advances in Cryogenic Engineering (Materials), vol. 40, edited by R. P. Reed et al, Plenum Press, New York, 1994, pp. 1383-1390.

4. J. Knobloch, "The "Q Disease in Superconducting Niobium RF Cavities" in Hydrogen in Materials \& Vacuum Systems”, edited by G. R. Myneni et al., AIP Conference Proceedings 671, American Institute of Physics, Melville, NY, 2003, pp. 133-150.

5. P. Kneisel, "Superconducting Radio Frequency Technology: An Overview", Materiaux \& Techniques vol 7-8-9, 2003 pp 7-12

6. G.R. Myneni and S.R. Agnew, "Elasto-Plastic Behavior of High RRR Niobium: Effects of Crystallographic Texture, Microstructure and Hydrogen Concentration” in Hydrogen in Materials and Vacuum Systems, edited by G. Myneni et al, AIP Conference Proceedings 671, Melville, New York, 2003, pp. 227-242

7. S Agnew, F Zeng, G.R. Myneni, Ultrasonic Velocity and Texture of High RRR Niobium” in Materiaux \& Techniques, vol 7-8-9, 2003 pp. 38-44

8. G. Myneni,H. Umezawa "Variation of Mechanical Properties of High RRR And Reactor Grade Niobium With Heat Treatments” in Materiaux \& Techniques, vol 7-8-9, 2003 pp. 19- 22

9. G. R. Myneni, P. Kneisel and T. Carneiro, JLab “CEBAF Invention Disclosure 1163”, 9-3-2004

10. G. R. Myneni, “Optimization of RRR Niobium Bulk Properties” in Proceedings of the workshop on Pushing the Limits of RF Superconductivity, edited by K-J Kim et al, ANL, 2005, pp. 333-338

11. G. R. Myneni. "Physical and Mechanical Properties of Single and Large Crystal High-RRR Niobium” in Proceedings of $12^{\text {th }}$ International Workshop on RF Superconductivity (SRF 2005), July 10 - 15, Ithaca, USA

12. G. R. Myneni et al, "The Influence of Hydrogen on the Performance Characteristics of SRF Cavities” to be published 